\title{
High power breakdown testing of a photonic band-gap accelerator structure with elliptical rods
}

\author{
Brian J. Munroe, ${ }^{*}$ Alan M. Cook, Michael A. Shapiro, and Richard J. Temkin \\ Plasma Science and Fusion Center, Massachusetts Institute of Technology, Cambridge, Massachusetts 02139, USA \\ Valery A. Dolgashev, Lisa L. Laurent, James R. Lewandowski, A. Dian Yeremian, and Sami G. Tantawi \\ SLAC National Accelerator Laboratory, Menlo Park, California 94025, USA \\ Roark A. Marsh \\ Lawrence Livermore National Laboratory, Livermore, California 94550, USA
}

(Received 30 August 2012; published 29 January 2013)

\begin{abstract}
An improved single-cell photonic band-gap (PBG) structure with an inner row of elliptical rods (PBG-E) was tested with high power at a $60 \mathrm{~Hz}$ repetition rate at X-band $(11.424 \mathrm{GHz})$, achieving a gradient of $128 \mathrm{MV} / \mathrm{m}$ at a breakdown probability of $3.6 \times 10^{-3}$ per pulse per meter at a pulse length of $150 \mathrm{~ns}$. The tested standing-wave structure was a single high-gradient cell with an inner row of elliptical rods and an outer row of round rods; the elliptical rods reduce the peak surface magnetic field by $20 \%$ and reduce the temperature rise of the rods during the pulse by several tens of degrees, while maintaining good damping and suppression of high order modes. When compared with a single-cell standing-wave undamped disk-loaded waveguide structure with the same iris geometry under test at the same conditions, the PBG-E structure yielded the same breakdown rate within measurement error. The PBG-E structure showed a greatly reduced breakdown rate compared with earlier tests of a PBG structure with round rods, presumably due to the reduced magnetic fields at the elliptical rods vs the fields at the round rods, as well as use of an improved testing methodology. A post-testing autopsy of the PBG-E structure showed some damage on the surfaces exposed to the highest surface magnetic and electric fields. Despite these changes in surface appearance, no significant change in the breakdown rate was observed in testing. These results demonstrate that PBG structures, when designed with reduced surface magnetic fields and operated to avoid extremely high pulsed heating, can operate at breakdown probabilities comparable to undamped disk-loaded waveguide structures and are thus viable for highgradient accelerator applications.
\end{abstract}

DOI: 10.1103/PhysRevSTAB.16.012005

PACS numbers: 29.20.Ej, 52.80.Pi, 52.80.Vp

\section{INTRODUCTION}

Photonic band-gap (PBG) structures continue to be a topic of experimental and theoretical interest in accelerator structure design [1-4]. Photonic crystals use a lattice of metallic or dielectric rods to prevent propagation of electromagnetic waves through the lattice at certain frequencies $[5,6]$. In accelerator applications this allows for a drive mode to be confined by a defect region within the lattice while damping modes at both higher and lower frequencies. A metallic PBG lattice can be designed such that the lowest frequency mode supported by the cavity is the drive mode and all higher order modes (HOMs) are damped. The initial design of a PBG accelerator structure was based on a square lattice as proposed in [7]. A triangular lattice provides better symmetry for accelerator structures, and good

\footnotetext{
*bmunroe@mit.edu

Published by the American Physical Society under the terms of the Creative Commons Attribution 3.0 License. Further distribution of this work must maintain attribution to the author(s) and the published article's title, journal citation, and DOI.
}

agreement with simulation has been shown for a triangular lattice in an accelerator application using a vector network analyzer (VNA) [8]. A $17 \mathrm{GHz}$, six-cell traveling wave PBG structure based on this triangular lattice was built [9] and tested at MIT, demonstrating acceleration [1]. PBG HOMs have been simulated and the wakefields have been measured $[4,10,11]$. A standing-wave high-gradient PBG structure has been tested for breakdown performance at SLAC [3].

An improved PBG design was made based on results of this initial high-gradient, high repetition rate testing of a PBG structure [12]. This design changes the shape of the rods immediately surrounding the defect region to reduce the peak surface magnetic field in the structure; this reduces pulsed heating and cyclic fatigue, which should improve structure performance. The improved lattice has been incorporated into a single-cell standing-wave structure for high-gradient high repetition rate testing at SLAC, a model of which is depicted in Fig. 1. This structure follows the general design used extensively in previous SLAC singlecell standing-wave structure testing [13-21]. The structure is designed with a matching cell on either side of a single 


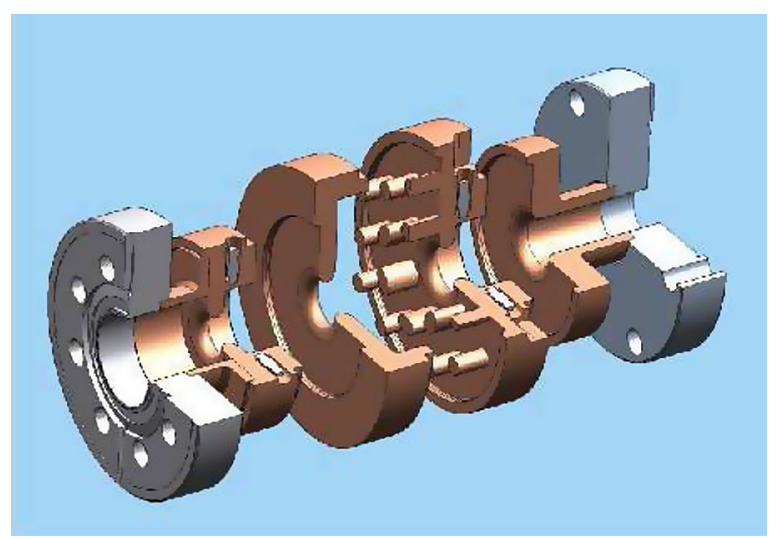

FIG. 1. Expanded three-quarter view of solid model of elliptical-rod PBG structure, showing two coupling cells and central PBG cell. Power is coupled in from the left.

PBG test cell. The structure is designed to have the highest electric and magnetic fields in the test cell and significantly reduced fields in the matching cells. The matching cells have solid cylindrical walls instead of PBG rods. The structure is axially powered via a reusable $\mathrm{TM}_{01}$ mode launcher [22], which remains with the structure for the duration of all cold and hot tests; this mode launcher design has been thoroughly tested in previous experiments.

\section{DESIGN OF PHOTONIC BAND-GAP (PBG) STRUCTURE}

The structure was designed to be directly comparable to the original high-gradient PBG structure, SLAC designation $1 \mathrm{C}-\mathrm{SW}-\mathrm{A} 5.65-\mathrm{T} 4.6-\mathrm{PBG}-\mathrm{Cu}$ and referred to here as a PBG structure with round rods (PBG-R) [3], and a disk-loaded waveguide structure fabricated at INFNFrascati and tested at SLAC [23], SLAC designation 1C-SW-A5.65-T4.6-Cu, referred to here as disk-loaded waveguide (DLWG). The SLAC designations indicate a single high-gradient cell, 1C, standing-wave (SW) structure with an iris aperture of $5.65 \mathrm{~mm}, \mathrm{~A} 5.65$, and iris thickness of $4.6 \mathrm{~mm}$, T4.6, made out of copper $(\mathrm{Cu})$. To this end, all three structures have irises with elliptical cross sections to reduce the peak surface electric field, and the same iris aperture and thickness for the irises on either side of the high-field cell. With these parameters fixed, the PBG cell parameters, the aperture of the coupling iris, and the radii of the coupling cells were left as design variables.

\section{A. PBG cell design}

In the three-cell design used for this and previous highgradient structure testing (e.g. [19]), the central cell is designed to have twice the on-axis electric field magnitude of the coupling cells on either side. This requires a PBG cell which strongly confines a $\mathrm{TM}_{01}$ accelerating mode at a frequency of $11.424 \mathrm{GHz}$. Critical coupling into the entire structure is also required.

The PBG structure with an inner row of elliptical rods (PBG-E) lattice has two main variations when compared to the lattice of the PBG-R structure. The most significant variation is the use of elliptical rods for the inner row of the structure, keeping the outer rods circular. Making the rods elliptical reduces the peak surface magnetic field on the rod, thereby reducing peak surface temperature rise; the field is much lower on the outer rods, making modification of these rods unnecessary. The lattice used in the ellipticalrod structure is shown in Fig. 2. The same lattice parameter of $\alpha / \beta=0.18$ is used for both the PBG-E and PBG-R structures [3]. In the elliptical-rod lattice the minor radius of the elliptical rods is kept the same as the radii of the round rods. The major radius is chosen to be 1.5 times the minor radius. This ratio of major to minor radius for the elliptical rods represents a trade-off between increasing the ratio of peak surface electric field to peak surface magnetic field, a measure of peak surface temperature rise at a given gradient, and keeping the $Q$ value of the $\mathrm{TM}_{11}$ mode low, as reported in [12].

The other significant change in the PBG-E cell design is the use of only two rows of rods as opposed to the three rows used in the PBG-R structure, making fabrication easier. In the absence of an outer wall, the number of rows of rods in the structure determines the diffractive $Q$ value of the modes. This $Q$ increases with the number of rows of rods. When a solid outer wall is present, as is required for vacuum purposes in the SLAC design, the diffractive $Q$ also increases, allowing the use of two rows of rods without a decrease in total $Q$ of the mode. A minimum of two rows of rods is required for proper confinement of the fundamental mode.

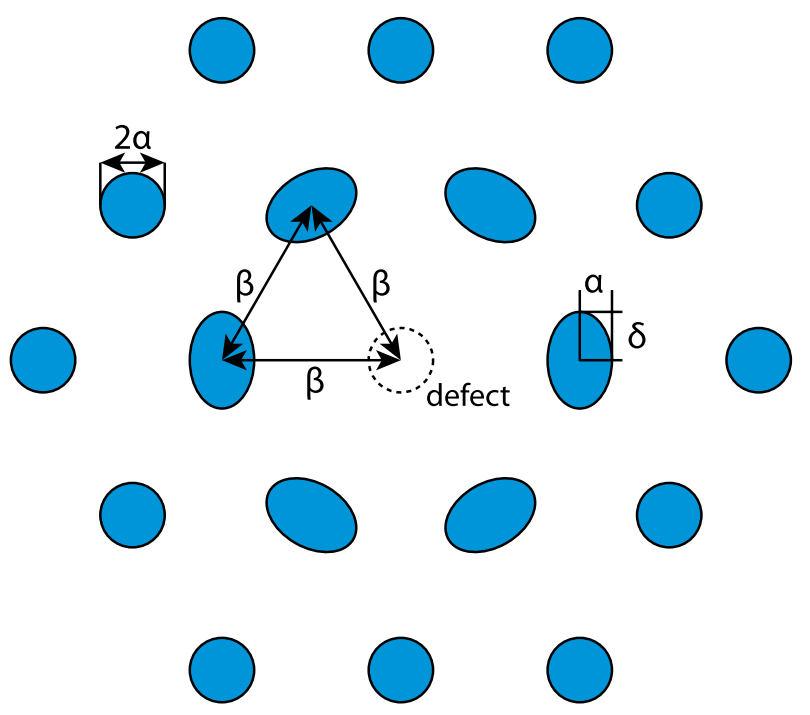

FIG. 2. Elliptical-rod PBG lattice showing lattice spacing $\beta$, rod radius (minor radius for elliptical rods) $\alpha$, and major radius $\delta$. 
TABLE I. Fixed parameters for PBG-E structure. Final design values for Fig. 3(a).

\begin{tabular}{cc}
\hline \hline \multicolumn{3}{c}{ Fixed parameters } \\
\hline Rpipe & $3.0 \mathrm{~mm}$ \\
Rb & $1.0 \mathrm{~mm}$ \\
$\mathrm{t}$ & $4.6 \mathrm{~mm}$ \\
e_r & $3.4 \mathrm{~mm}$ \\
$\mathrm{D}$ & $13.12 \mathrm{~mm}$ \\
$\mathrm{a}$ & $5.65 \mathrm{~mm}$ \\
a_pipe & $6.35 \mathrm{~mm}$ \\
b_conv & $11.43 \mathrm{~mm}$ \\
\hline \hline
\end{tabular}

\section{B. Structure tuning}

In addition to the iris geometry, various other structure parameters are fixed by the frequency of the experiment and the geometry of the SLAC mode launchers being used. These parameters are shown in an axisymmetric view, showing the vacuum space of the structure, in Fig. 3(a), and listed in Table I.

The tuning parameters of the structure are shown in Fig. 3(b). The radii of the input and output coupling cells, b_cpl and b_end, respectively, are varied in HFSS simulations to find a field profile on axis with approximately half of the peak field amplitude in the coupling cells relative to the central PBG cell. The radius to the outer wall of the PBG cell, b_cll, is chosen to allow space between the outer row of rods and the outer wall of the vacuum can. The aperture of the coupling iris, a_cpl, is chosen to achieve critical coupling in the HFSS simulation. All three parameters a_cpl, b_cpl, and b_end, affect the frequency of the structure, and must be adjusted to keep the resonant
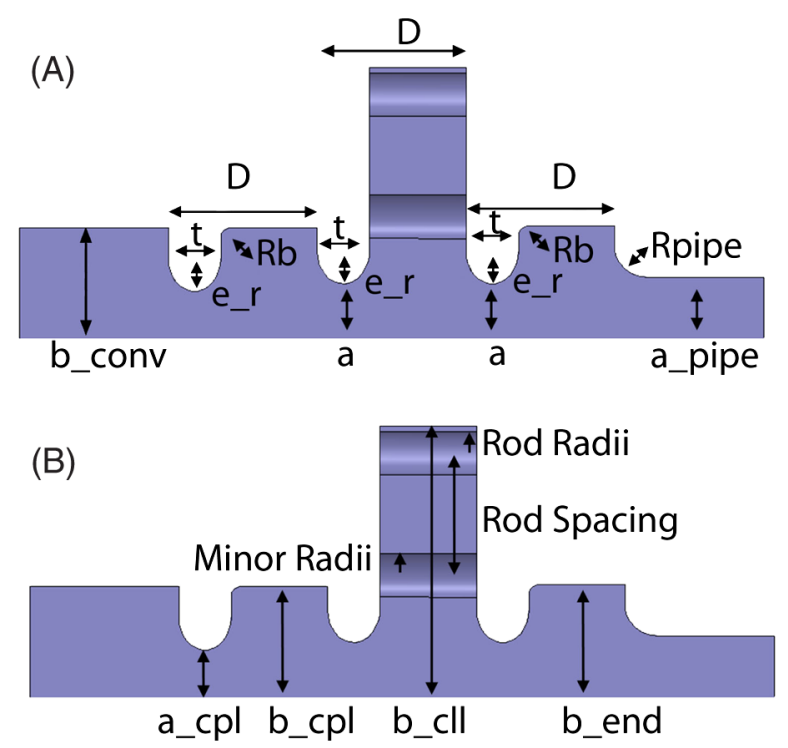

FIG. 3. Axisymmetric views of the vacuum space of the PBGE structure showing the fixed parameters (a), and tuning parameters (b), for the elliptical-rod PBG structure.
TABLE II. Tuning parameters for PBG-E structure. Final design values for Fig. 3(b).

\begin{tabular}{cr}
\hline \multicolumn{3}{c}{ Tuning parameters } \\
\hline a_cpl & $4.83 \mathrm{~mm}$ \\
b_cpl & $11.44 \mathrm{~mm}$ \\
b_cll & $28.0 \mathrm{~mm}$ \\
b_end & $11.63 \mathrm{~mm}$ \\
Minor radii $(\alpha)$ & $2.27 \mathrm{~mm}$ \\
Major radii $(\delta)$ & $3.40 \mathrm{~mm}$ \\
Rod spacing $(\beta)$ & $12.59 \mathrm{~mm}$ \\
\hline \hline
\end{tabular}

frequency of the entire structure as close as possible to the resonant frequency of the PBG cell, which is tuned to the center frequency of the klystron, $11.424 \mathrm{GHz}$. The final design values for the tuning parameters are given in Table II.

\section{Design results}

The elliptical-rod PBG test structure, PBG-E, was designed using HFSS [24]. The field amplitude on axis for the structure is shown in Fig. 4, showing the 1:2:1 relationship between the field amplitudes in the cells. The reflection from the cavity as a function of frequency $\left(S_{11}\right)$ is shown in Fig. 5, showing that a $\pi$ mode minimum reflection of $-49 \mathrm{~dB}$ is achieved at $11.427 \mathrm{GHz}$; this is sufficiently close to the center frequency of the klystron for testing.

Field plots for the final design are shown in Figs. 6 and 7 in a cutaway view through an inner rod and top-down view, respectively. In the cutaway view the 1:2:1 relationship in electric field value can be seen, as well as the increase in surface electric field on the irises. This view also shows the localization of the magnetic field to the defect-facing side of the inner rods. The top-down view shows the uniformity of the accelerating field in the defect region, and the distribution of the magnetic field across the defect-facing side of the inner rod.

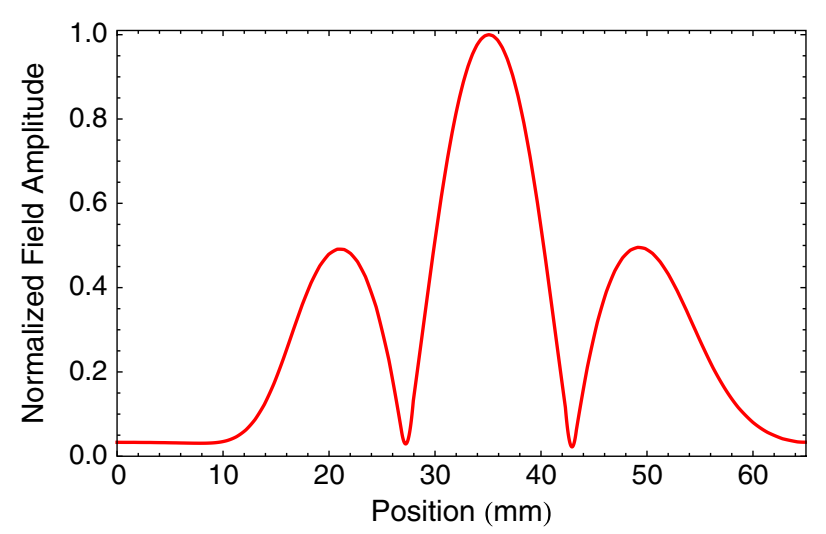

FIG. 4. Normalized electric field profile on axis in PBG-E structure; the field amplitude in each coupling cell is approximately half that in the PBG cell. 


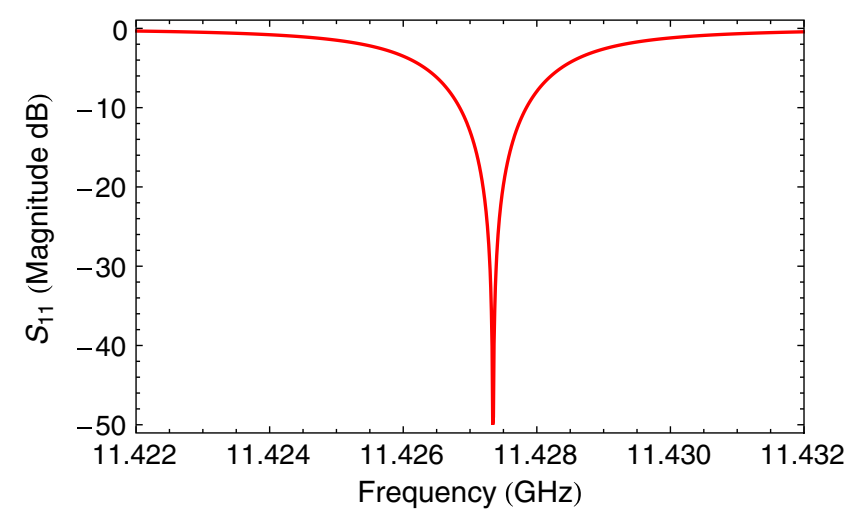

FIG. 5. Calculated reflection as a function of frequency for the PBG-E structure.
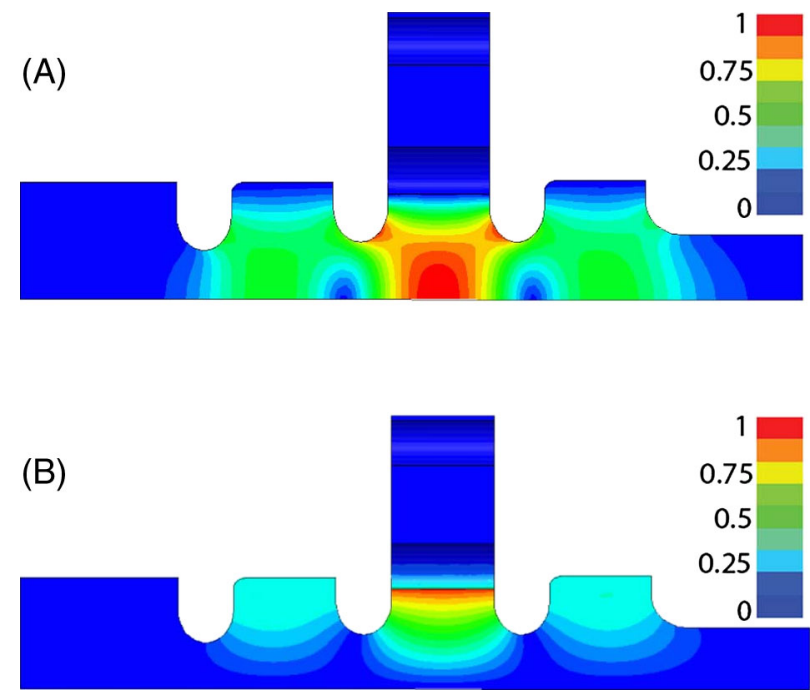

FIG. 6. Electric (a) and magnetic (b) field amplitudes looking at a radial cut of the structure through an inner rod. At an accelerating gradient of $100 \mathrm{MV} / \mathrm{m}$ the peak surface electric field amplitude is $207 \mathrm{MV} / \mathrm{m}$ at the iris surfaces. At the same gradient the peak surface magnetic field occurs on the inner surface of the inner rod, and reaches a value of $713 \mathrm{kA} / \mathrm{m}$.

\section{HFSS simulations for data analysis}

The data collected during experimental operation is in the form of number of breakdowns versus power incident on the structure. In order to translate this data into breakdown probabilities as a function of field parameters, such as gradient or peak surface temperature rise, calibrations between input power and field must be made. This can be done using driven simulations in HFSS, which allow the user to specify the frequency or frequencies of the simulation as well as the input power at the input port. To calculate the gradient and shunt impedance an eigenmode model of the central PBG cell is simulated with a phase advance of 180 degrees across the cell. The integrated gradient in this single-cell model is calculated as a fraction of the peak axial electric field. This relationship between

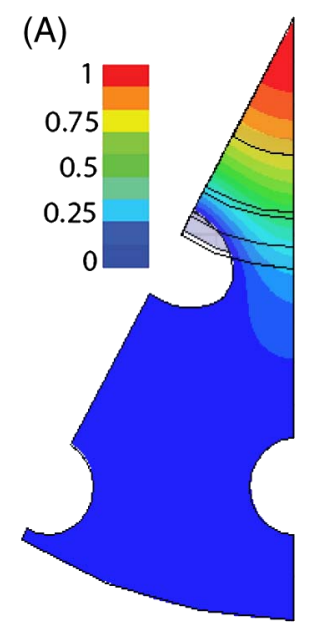

(B)

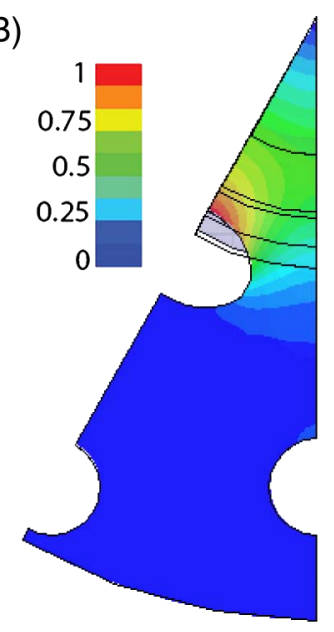

FIG. 7. Electric (a) and magnetic (b) field amplitudes looking at a top-down view of the structure. The peak surface electric field of $207 \mathrm{MV} / \mathrm{m}$ for a $100 \mathrm{MV} / \mathrm{m}$ accelerating gradient is seen on axis. The peak surface magnetic field of $713 \mathrm{kA} / \mathrm{m}$ for an accelerating gradient of $100 \mathrm{MV} / \mathrm{m}$ is confined to the center of the inner surface of the innermost rod.

gradient and peak surface field is used to provide a calibration between input power and effective gradient in the three-cell structure; this calibration is used in the analysis of the experimental data. For the elliptical-rod PBG structure the structure achieves $100 \mathrm{MV} / \mathrm{m}$ gradient at 4.4 MW. The fields in the structure scale as the square root of the input power, so this result can be used to find the field values at any input power. The surface fields at $100 \mathrm{MV} / \mathrm{m}$ gradient for both PBG structures and the equivalent disk-loaded waveguide structure are given in Table III.

\section{E. Surface temperature rise calculation}

The elliptical-rod PBG structure was designed with a goal of reducing the peak surface magnetic field, which causes surface temperature rise via Ohmic heating. This heating cannot be measured experimentally, but can be predicted mathematically using Eq. (1) [25]:

TABLE III. Surface field values from HFSS simulations for PBG-E structure, PBG-R structure, and disk-loaded waveguide structure. The shunt impedance calculated here is for a periodic $\pi$ mode structure; i.e., a structure composed of iterations of the central cell of the three-cell structures tested.

\begin{tabular}{lccc}
\hline \hline Structure & PBG-E & PBG-R & DLWG \\
\hline Power & $4.4 \mathrm{MW}$ & $5.9 \mathrm{MW}$ & $3.9 \mathrm{MW}$ \\
Gradient & $100 \mathrm{MV} / \mathrm{m}$ & $100 \mathrm{MV} / \mathrm{m}$ & $100 \mathrm{MV} / \mathrm{m}$ \\
$Q_{0}($ measured) & 7792 & 7401 & 8870 \\
$r_{s h}$ & $37 \mathrm{M} \Omega / \mathrm{m}$ & $36 \mathrm{M} \Omega / \mathrm{m}$ & $51 \mathrm{M} \Omega / \mathrm{m}$ \\
Peak surface $E$ field & $207 \mathrm{MV} / \mathrm{m}$ & $208 \mathrm{MV} / \mathrm{m}$ & $211 \mathrm{MV} / \mathrm{m}$ \\
Peak surface $H$ field & $713 \mathrm{kA} / \mathrm{m}$ & $890 \mathrm{kA} / \mathrm{m}$ & $418 \mathrm{kA} / \mathrm{m}$ \\
\hline \hline
\end{tabular}


TABLE IV. Calculated peak surface temperature rise for both PBG structures at $100 \mathrm{MV} / \mathrm{m}$ accelerating gradient.

\begin{tabular}{lc}
\hline \hline Structure & Temperature rise $(\mathrm{K})$ \\
\hline PBG-R & 131 \\
PBG-E & 84 \\
\hline \hline
\end{tabular}

$$
\Delta T=\frac{1}{\rho c_{\epsilon} \sqrt{\pi \alpha_{d}}} \int_{0}^{t} \frac{1}{\sqrt{t-t^{\prime}}} \frac{1}{2} R_{S}\left|H\left(t^{\prime}\right)\right|^{2} d t^{\prime} .
$$

Equation (1) relates the surface temperature rise $\Delta T$ to the surface magnetic field as a function of time $H\left(t^{\prime}\right)$, using the density $\rho$, specific heat at constant strain $c_{\epsilon}$, thermal diffusivity $\alpha_{d}$, and surface resistance $R_{S}$. This equation can be used with a model of the real pulse shape to predict the temperature rise prior to high-power testing, which can be used to limit the power applied to the structure. A comparison of the calculated peak surface temperature rise for the two PBG structures, given a pulse with a $180 \mathrm{~ns}$ filling portion and $150 \mathrm{~ns}$ flattop at $100 \mathrm{MV} / \mathrm{m}$ gradient is given in Table IV.

\section{COLD TEST}

The initial cold test and final tuning of the elliptical-rod PBG structure were done using the same $\mathrm{TM}_{01}$ mode launcher used for the high-power testing and postoperation cold test. A vector network analyzer (VNA) was connected to the rectangular waveguide input of the launcher and used to measure the complex reflection from the structure. All cold testing was done with a dry nitrogen flow through the structure to maintain structure cleanliness. The temperature of the structure was monitored via a thermocouple attached to the structure body.

The structure is tuned via four tuning studs in each matching cell, allowing the radius of the cells to be perturbed. The structure is tuned to produce the desired 1:2:1 field profile in addition to minimizing the $S_{11}$ of the $\pi$ mode. The final observed frequency of the $\pi$ mode of the structure is $11.440 \mathrm{GHz}$, which is within the acceptable tuning range of the klystron. This frequency shift is likely due to a large braze fillet around the rod ends, which was introduced to correct problems with the rod braze joints in the PBG-R structure; this large fillet is visualized in the autopsy of the structure.

After the structure is tuned the field profile is measured using a nonresonant perturbation technique [26-28]. The perturbing object is a small dielectric "bead" on a thin dielectric wire, which is suspended in the structure via a pulley assembly mounted to the top of the structure. The wire used is Ashaway 10/0 black monofilament microsuture thread and the bead is a small drop of super glue.

\section{A. Bead pull measurements}

The PBG structure supports multiple modes depending on the phase advance between the cells. The frequencies of

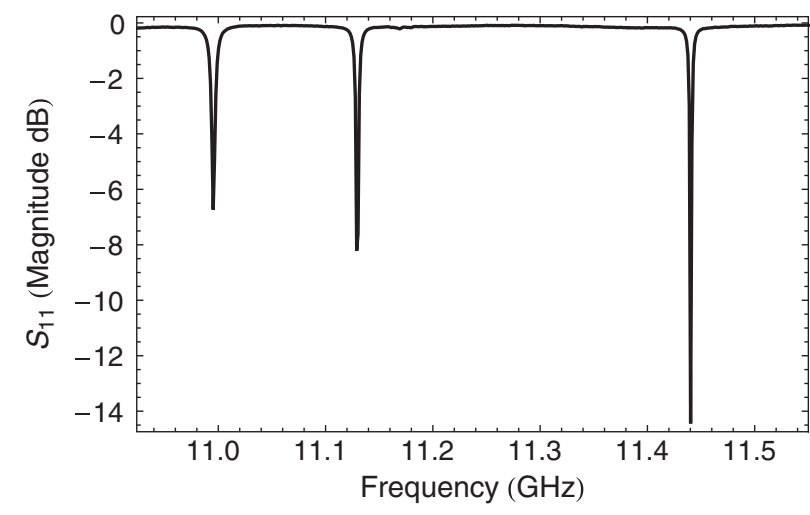

FIG. 8. $S_{11}$ of PBG-E structure, showing resonances for, in order of increasing frequency, the 0 mode, the $\pi / 2$ mode, and the $\pi$ mode.

these modes can be found by measuring the $S_{11}$ of the structure in the absence of any perturbing elements, as seen in Fig. 8. The modes can be identified by their field patterns, which are found by moving the bead through the full length of the structure and measuring the real and imaginary reflection on or near resonance as a function of axial distance.

Three structure modes were identified, the 0 mode, the $\pi / 2$ mode, and the $\pi$ mode. The structure is designed to operate in the $\pi$ mode, so it is of primary importance for the axial field profile of this mode to match the design. Good agreement between the design and measured field was found in the test and matching cells as shown in Fig. 9.

\section{B. Coupling and $Q$}

Using the $S_{11}$ measurement, the resonant frequency, coupling, and mode $Q$ values can be determined for each structure mode [29]. The unloaded $Q, Q_{0}$, and external $Q$, $Q_{\text {ext }}$, can be measured directly from the Smith chart. These are then used to calculate the loaded $Q, Q_{L}$, via $1 / Q_{L}=$ $1 / Q_{0}+1 / Q_{\text {ext }}$. These values are summarized in Table V.

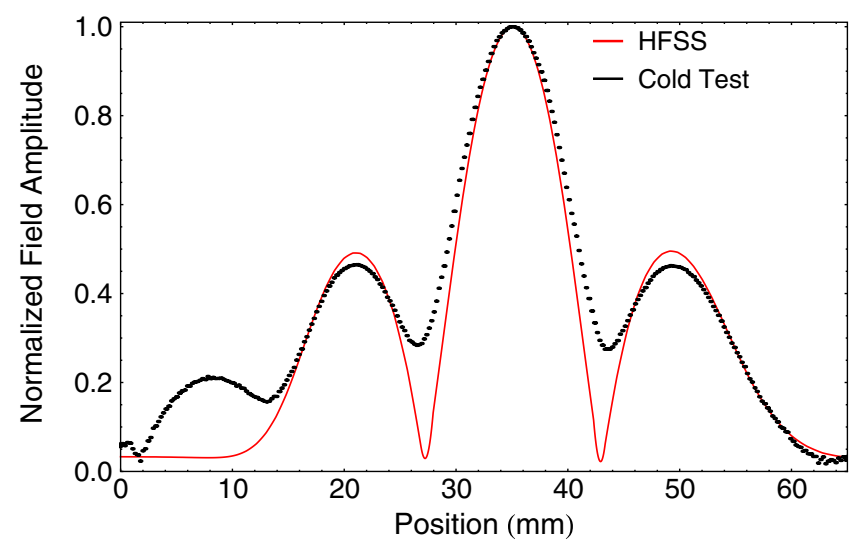

FIG. 9. $\pi$ mode field profile 
TABLE V. $Q$ values for the various structure modes.

\begin{tabular}{lcccc}
\hline \hline & & & $Q$ & \\
Mode & Frequency $(\mathrm{GHz})$ & $Q_{0}$ & $Q_{\mathrm{ext}}$ & $Q_{L}$ \\
\hline 0 & 10.995 & 9057 & 3058 & 2286 \\
$\pi / 2$ & 11.129 & 9319 & 5304 & 3380 \\
$\pi$ & 11.440 & 7792 & 10290 & 4434 \\
\hline \hline
\end{tabular}

\section{EXPERIMENTAL SETUP}

The PBG structure was tested at the SLAC National Accelerator Laboratory, using the X-band klystron XL4-6B. This klystron is controlled by a computer system, which allows the use of shaped pulses as well as controlling the power and frequency of the rf on a shot to shot basis. The power at the klystron is measured with an Agilent 8990 A peak power meter, and the power incident on and reflected by the structure is measured with an Agilent $\mathrm{N}-1912 \mathrm{~A}$ peak power meter; traces from both power meters are recorded every two seconds. The filling of the standingwave structure is done using a shaped pulse with a higherpower portion filling the structure rapidly, after which the power decreases to maintain a constant power coupled into the structure for the duration of the pulse; the quoted pulse power and pulse length reflect this constant-power portion of the pulse. The control system monitors the power and frequency of the applied rf and makes changes as needed. The structure is cooled via external water flow in a copper jacket which keeps the frequency shift at the few megahertz level, well within the bandwidth of the klystron. In addition to the peak power meters, there are crystal detectors on both the forward and reflected power signals, current monitors measuring the current in each direction, and an ion gauge measuring structure pressure. A log is kept for the test stand, providing records of the operating conditions and testing goals for the current experiment.

During testing two distinct sets of data are recorded by microwave diagnostics. The peak power meters record the klystron, forward, and reflected power every two seconds while the system is operating. The current monitor signals are measured by an oscilloscope and used to determine when a breakdown has occurred. When a breakdown is detected, based on the magnitude of the current monitor signal, the forward power crystal signal, reflected power crystal signal, forward current monitor signal, and reverse current monitor signal are recorded for that trace and the trace immediately before the breakdown was detected. This provides a fast way of counting the number of breakdowns during testing, and the forward and reverse crystal signals provide a monitor of the microwave pulses.

\section{TESTING METHODOLOGY}

\section{A. Experience with first PBG structure}

A PBG structure with only round rods, PBG-R, was tested at SLAC with the standard SLAC testing methodology, as reported in [3]. The goal of this standard methodology is to collect systematic and reproducible data as fast as is practical. To this end the structures are typically exposed to very high power at low pulse length early in the testing. This produces a large number of breakdowns in a short time, and is intended to process the surface in a minimal amount of time. After this initial processing phase, in which many hundreds or thousands of breakdowns may occur, the structure is operated at lower breakdown rates, with the goal of producing data sets which show a linear relationship between accumulated breakdowns and time. By taking data at different power levels and pulse lengths it is possible to generate plots showing breakdown probability as a function of gradient or pulsed heating. This methodology has been applied to many disk-loaded waveguide-type structures and produced repeatable results showing no degradation in structure performance during testing. Use of this protocol with a structure with very high peak surface temperature rise, such as the PBG-R structure, may have degraded the performance of the structure very early in the testing.

Contrary to previous tests of conventional structures, the post-testing autopsy of the PBG-R structure showed no damage at high electric field locations. Damage was instead seen at high magnetic field locations. This indicates that the breakdowns that occurred in the PBG-R structure were qualitatively different from those seen in previous high-gradient structure tests conducted at SLAC. It is therefore likely that use of the testing methodology normally used at SLAC, which results in the accumulation of a large number of breakdowns early in the testing, caused an irrevocable degradation of the performance of the PBG-R structure very early in the testing.

Another possible source of degraded performance in the PBG-R structure is the poor braze connection between the rods and the copper end plate, as seen in post-testing scanning electron microscope (SEM) imaging. This braze joint resulted in a negative curvature region at the brazed end of the rod (as opposed to a positive-curvature fillet as intended). Such regions can increase the breakdown probability in several ways, including trapping pockets of gas and supporting multipactoring. Because the damage to the PBG-R rods looks uniform along the length of the rod, it is unlikely that the poor braze joint is the sole cause of the decreased performance observed in the PBG-R structure, although it may have contributed. The brazing technique was modified for the PBG-E structure to avoid negative curvature regions, resulting in very large fillets at the brazed end of the rods.

The hypothesis that the PBG-R structure was damaged early in testing is supported by calculated pulsed heating temperature rise for the PBG-R structure (Fig. 10), indicating extremely high surface temperature rises well above the "safe" limit of $50 \mathrm{~K}$ [30] early in the processing.

\section{B. Revised testing methodology}

The goal of the revised testing methodology was to show that PBG-type structures can operate at high gradients and 


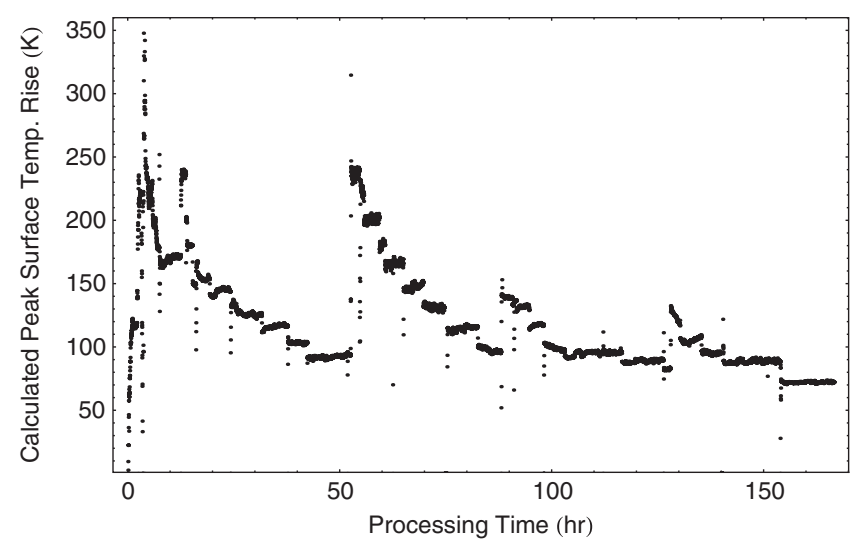

FIG. 10. Calculated peak surface temperature rise due to pulsed heating in the PBG-R structure. Note the excursions to over $200 \mathrm{~K}$ early in the testing.

low breakdown probability simultaneously. This means that any performance degradation early in testing, as may have occurred in the PBG-R testing, should be avoided. This was done by using a testing methodology for the PBG-E structure that limited both the calculated pulsed heating and the breakdown rate. The calculated pulsed heating was limited to $150 \mathrm{~K}$ and the breakdown rate was limited to a steady-state rate of 10 per hour. This methodology better protects the structure from damage, but significantly increases the duration of the testing, because a comparable number of breakdowns must be accumulated for each structure. Because of power level and performance fluctuations on a day-to-day basis, each breakdown probability must be calculated from a single day. Each day is limited to a run time of approximately 14 hours, and approximately ten breakdowns are needed in a data set to achieve reasonable error bars. This limits the practical breakdown rate to a rate of at least 1 per hour. The imposed upper limit of ten breakdowns per hour for structure protection means that data can only be taken over 1 order of magnitude in breakdown probability; this is a necessary and acceptable consequence of protecting the structure.

\section{DATA ANALYSIS}

The critical data for analysis of the structure performance is stored in the oscilloscope and peak power meter traces; this data must be analyzed to determine the gradient and pulsed heating at which breakdowns occurred. Breakdowns are counted by looking at the current monitor channels of the scope data sets. Using the time stamps on these files, breakdowns can be correlated with precise power and pulse length information from the peak power meter data sets. The power level and pulse length from the peak power meter is then translated into structure fields using calibrated HFSS simulations. The analysis for both scope and peak power meter data was done using Wolfram MATHEMATICA [31]. Data from both the baseline disk- loaded waveguide structure (1C-SW-A5.65-T4.6-Cu, [17]) and the PBG-R structure (1C-SW-A5.65-T4.6-PBG$\mathrm{Cu}$, [3]) were reanalyzed using the same algorithms as the elliptical rod PBG (1C-SW-A5.65-T4.6-PBG2-SLAC-Cu) to ensure consistency between results.

\section{A. Scope traces}

The scope traces contain both uncalibrated forward and reflected power information and the forward and reverse current monitor signals. A sample set of scope traces showing a shot without a breakdown is shown in Fig. 11. The next shot, on which a breakdown occurred, is shown in Fig. 12; the breakdown can be identified by the spike in both current monitor signals. An increase in the reflected power can also be seen.

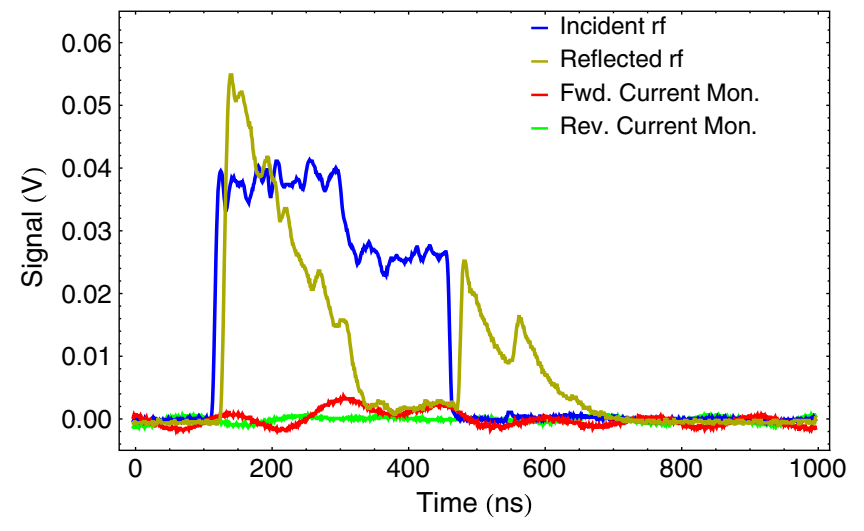

FIG. 11. A sample set of scope traces showing a normal shot. This is a $150 \mathrm{~ns}$ shot at approximately $125 \mathrm{MV} / \mathrm{m}$ gradient. The measured incident $\mathrm{rf}$ signal is shown in blue, and the measured reflected rf signal is shown in yellow. The forward and reverse current monitor signals are shown in red and green, respectively.

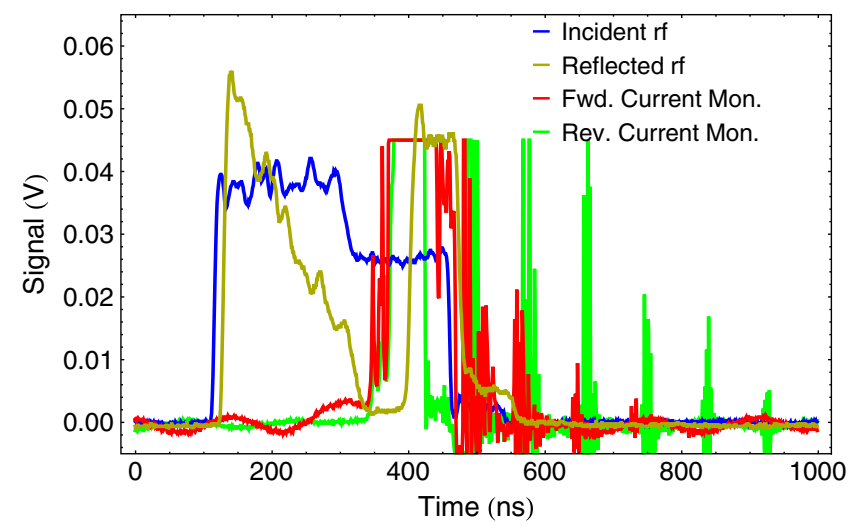

FIG. 12. A sample set of scope traces showing a breakdown shot, as indicated by the peaks in the current monitor signals, and an increase in reflected $\mathrm{rf}$ signal. This is a $150 \mathrm{~ns}$ shot at approximately $125 \mathrm{MV} / \mathrm{m}$ gradient. The measured incident power is shown in blue, and the measured reflected power is shown in yellow. The forward and reverse current monitor signals are shown in red and green, respectively. Note that the currents generated by the breakdowns saturate the current monitors. 
The computer system records every shot where there is a breakdown, as well as the shot before, triggered based on the level of the current monitor signals. This means that in post-processing the breakdown shots must be separated from the nonbreakdown shots to allow for accurate counting of the accumulated breakdowns. This is done using a MATHEMATICA program which generates a set of data containing the time for each breakdown and the accumulated number of breakdowns for all the testing up to that time. Because the system fires at $60 \mathrm{~Hz}$, secondary breakdowns, i.e., breakdown shots immediately preceded by a breakdown shot instead of a normal shot, can be identified by looking at the time interval between breakdowns.

\section{B. Peak power meter traces}

All three peak power meter signals are recorded, but in practice only the forward power channel is used in data analysis. During data analysis the reflected power can be used to check that the system was on resonance for any given forward power meter trace, but this is not generally necessary as the control system is able to quickly find the resonant frequency as it varies and maintain resonance throughout the day. These traces, as well as the power coupled into and reflected by the cavity, are shown in Fig. 13.

For each forward power trace, the real system response for that input signal is calculated using the $S_{11}$ values obtained in the cold test. This gives an actual power coupled into the structure for each pulse, which is turned into actual fields and then actual gradient and pulsed heating measurements using HFSS simulations. The measured input rf trace, calculated gradient, and calculated peak surface temperature rise are shown in Fig. 14. The actual input power and gradient are then averaged over the flattop of the pulse, which is calculated for each trace using the known duration of the filling portion of the pulse. The

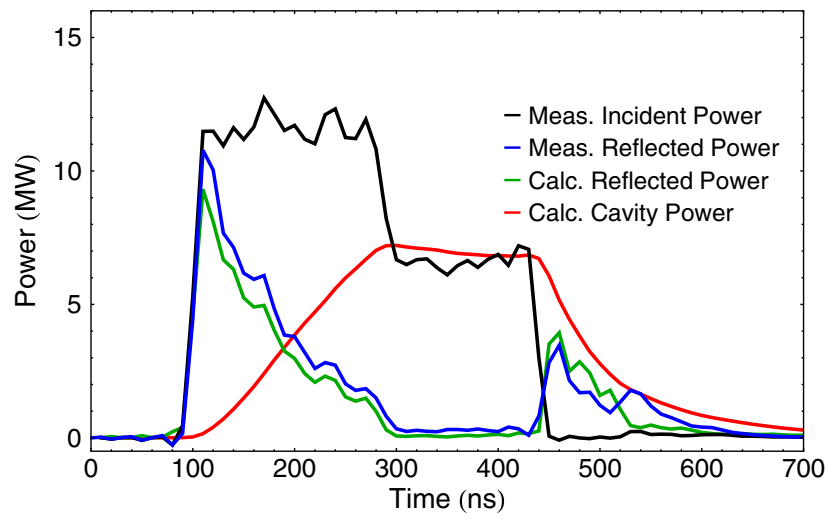

FIG. 13. Sample traces from the peak power meter for a $150 \mathrm{~ns}$ pulse at a gradient of $126 \mathrm{MV} / \mathrm{m}$. The incident and reflected power measured by the peak power meter are shown in black and blue, respectively. The calculated reflected power and power coupled into the structure are shown in green and red, respectively.

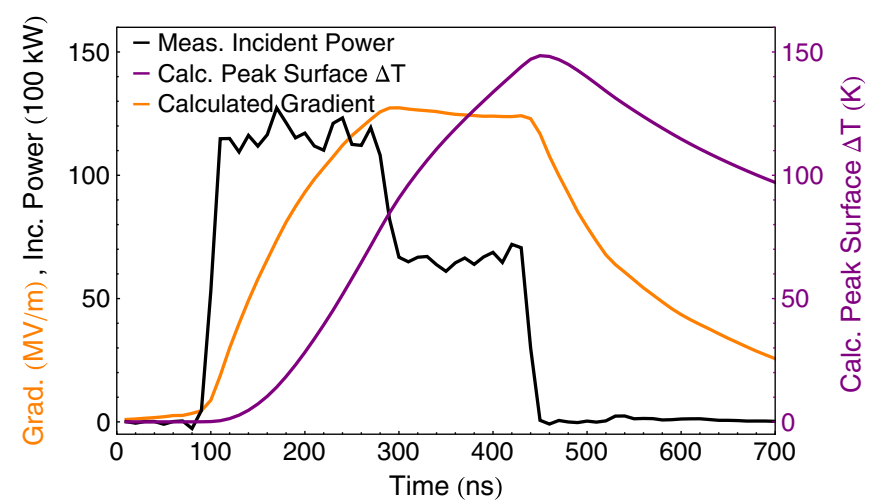

FIG. 14. Sample traces from the peak power meter for a $150 \mathrm{~ns}$ pulse at a gradient of $126 \mathrm{MV} / \mathrm{m}$. The incident power measured by the peak power meter is shown in black. The calculated gradient is shown in orange, and the calculated peak surface temperature rise is shown in purple.

time stamp, pulse length, average filling and flattop power, average gradient, and maximum value of pulsed heating are all recorded for each trace.

\section{Combining results}

The time stamps on the scope traces and peak power meter traces can be used to correlate the two data sets, providing a plot of accumulated breakdowns and gradient versus time, shown in Fig. 15. By looking for sections of these plots where the gradient is approximately constant and the number of breakdowns increases linearly with time, values of breakdown probability versus gradient can be found. Values of breakdown probability versus peak pulsed heating or field values can be found using a similar procedure. The structure was tested at high gradient for more than 350 hours.

A plot of the peak surface temperature rise for the duration of testing (Fig. 16) shows that a majority of the testing was done at greater than the safe temperature rise of $50 \mathrm{~K}$ [30], with many pulses showing a temperature rise between 100 and $150 \mathrm{~K}$. Pulses with a peak surface

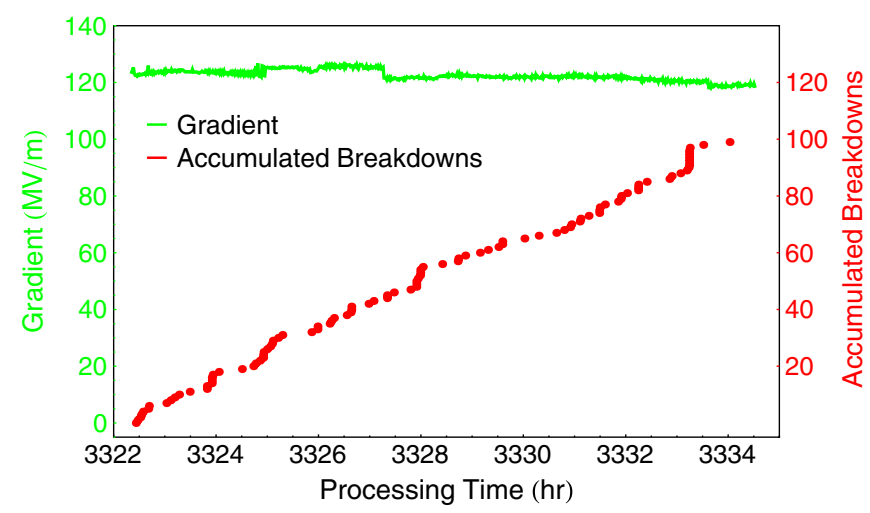

FIG. 15. Accumulated breakdowns and gradient during the majority of testing. 


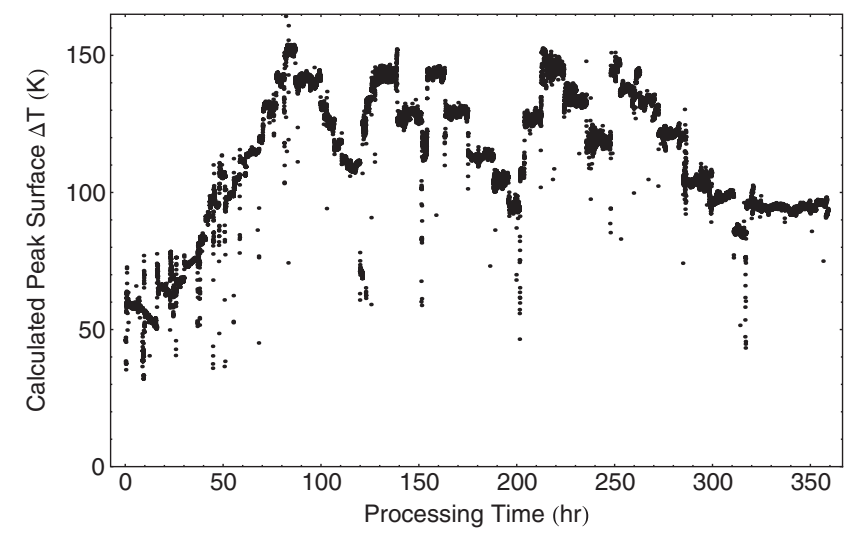

FIG. 16. Calculated peak surface temperature rise due to pulsed heating in the PBG-E structure.

temperature rise of less than approximately $100 \mathrm{~K}$ correspond to a breakdown rate less than the artificial lower limit of 1 per hour. This restriction, along with an effort to achieve the largest possible gradient, explains the higher average peak surface temperature rise.

\section{EXPERIMENTAL RESULTS}

For the elliptical-rod PBG structure breakdown probability measurements were made at pulse lengths of 150 , 200, 400, and $600 \mathrm{~ns}$. This spans a large portion of the phase space of gradient and peak pulsed heating, as well as providing comparisons to the previous structures. A comparison of breakdown probability versus gradient at a pulse length of $150 \mathrm{~ns}$ for all three structures is shown in Fig. 17. The two data sets for the second PBG structure represent the beginning (run 1) and end (run 2) of the testing, indicating no degradation in performance over the course of the testing. The data shows that the breakdown probability increases with the gradient in the structure for a fixed

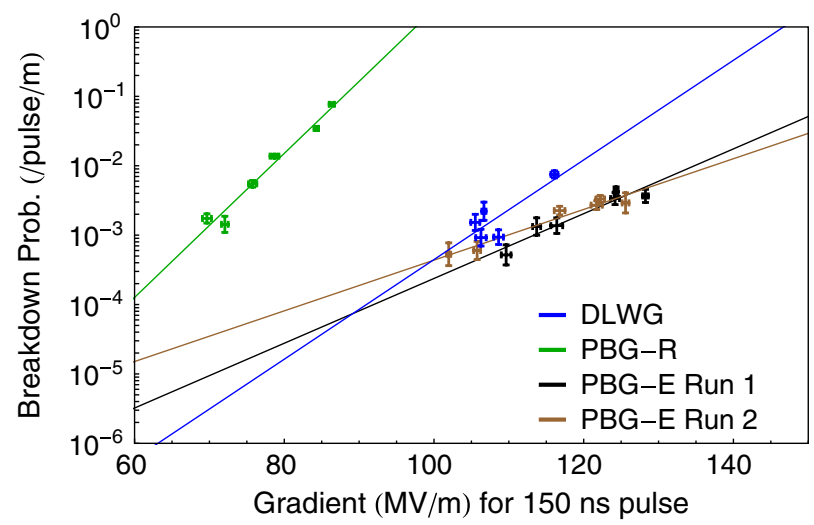

FIG. 17. Breakdown probability per pulse per meter of structure vs gradient at $150 \mathrm{~ns}$ pulse length for the PBG-R, PBG-E, and a conventional disk-loaded waveguide structure with the same iris geometry (DLWG). The two data sets for the PBG-E represent the beginning and end of testing. pulse length. All three structures have the same iris geometry and peak surface electric fields that are approximately twice the gradient. The variation in breakdown probability between the PBG-R and PBG-E is likely due to the damage sustained by the PBG-R structure early in testing. The significant improvement in performance between the two PBG structures is likely due in part to the improved design and fabrication and in part to the improved testing methodology, both of which contributed to protecting the PBG-E structure during testing. From Fig. 17 it appears that, for undamaged structures, the gradient provides a good prediction of the breakdown probability for the PBG-E and disk-loaded waveguide structures with the same iris geometry at the same pulse length; previous work at SLAC has shown that gradient is not a good predictor of breakdown probability for structures with different iris geometries [19]. More importantly, Fig. 17 indicates that the elliptical-rod PBG structure can operate at gradients and breakdown rates comparable to a disk-loaded waveguide structure, while simultaneously providing wakefield damping.

Comparison of the breakdown probability as a function of gradient at different pulse lengths in the PBG-E structure, as shown in Fig. 18, indicates that gradient is not sufficient to predict breakdown probability for the PBG-E structure at different pulse lengths, i.e., the breakdowns in the PBG-E structure are not purely due to electric field effects. Previous work with disk-loaded waveguide structures with different iris geometries suggests that the peak surface temperature rise is a good figure of merit for predicting the breakdown probability of a given structure at different pulse lengths, e.g. [18,19]. This is not the case in the PBG-E structure, as seen in Fig. 19. Neither gradient nor peak surface temperature rise alone is sufficient to predict the breakdown probability of the PBG-E structure, indicating that the breakdown probability in the PBG-E structure is likely the result of both electric and magnetic field effects.

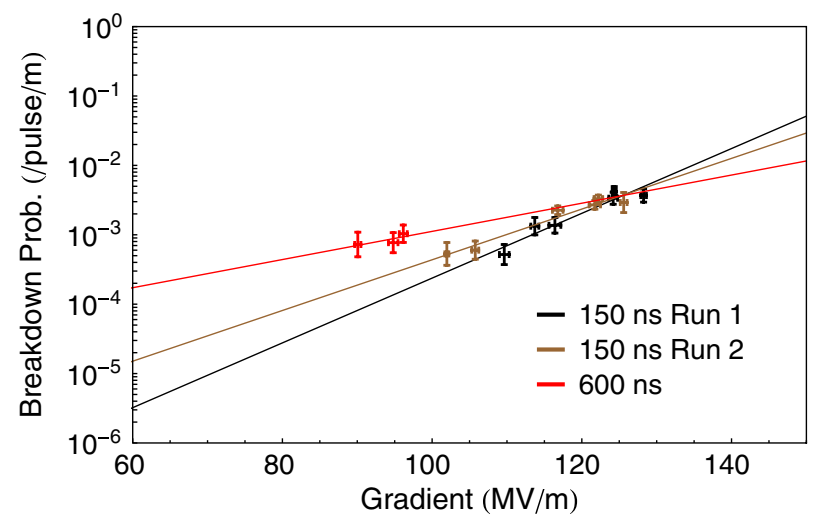

FIG. 18. Breakdown probability per pulse per meter of structure vs gradient for PBG-E at pulse lengths of 150 and 600 ns. 


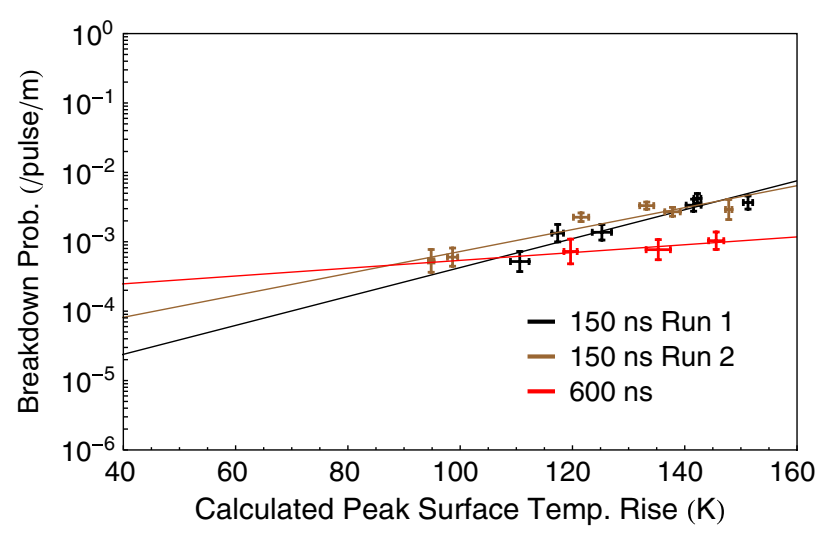

FIG. 19. Breakdown probability per pulse per meter of structure vs peak surface temperature rise for PBG-E at pulse lengths of 150 and $600 \mathrm{~ns}$.

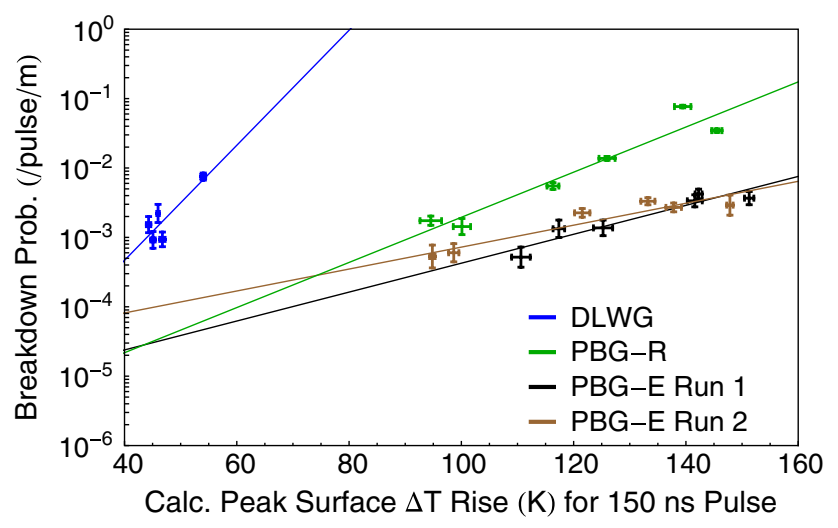

FIG. 20. Breakdown probability per pulse per meter of structure vs peak surface temperature rise for PBG-E, PBG-R, and DLWG structures at a pulse length of $150 \mathrm{~ns}$.

The breakdown probability for all three structures can also be plotted as a function of calculated peak surface temperature rise, shown in Fig. 20, which shows the breakdown probability at a pulse length of $150 \mathrm{~ns}$ for all three structures. This shows that the peak surface temperature rise is not sufficient to predict the breakdown probability across multiple structures, indicating that ratio of peak surface magnetic field to accelerating gradient, which varies between the three structures, plays a role in determining the breakdown probability. The elliptical-rod PBG does show marked improvement over the PBG-R structure at similar surface temperature rise, indicating that these temperatures are not sufficient to cause high breakdown probability, even when combined with high gradients. These temperatures occur at much higher gradients in the PBG-E structure than the PBG-R structure; this is a result of the improved design.

\section{AUTOPSY}

After high-power testing the structure was cold tested again. This indicated a decrease in $Q_{0}$ for the operating mode from 7800 to 7400 , a decrease of approximately 5\% from the original value. The structure was then cut in half and examined with a scanning electron microscope (SEM). The structure is cut along the longitudinal direction, with the plane of the cut passing through a rod in the second row but missing all of the rods in the inner row. Cutting along this plane allows SEM imaging of the high-field irises of the structure, on either side of the PBG cavity, and the PBG rods themselves; the input coupling iris and the outer rods of the PBG cavity can be taken as unprocessed surfaces due to the significantly lower fields in these regions.

SEM imaging of the high-field irises showed some damage to both sides of the irises, as seen in Fig. 21. This micrograph shows the input iris of the PBG cell, looking perpendicular to the axis of the structure, so that the curvature of the iris cannot be readily seen; the highfield side of the iris is on the right of the image. White splotches of breakdown damage can be seen, as well as grain growth due to pulsed heating. The larger particles visible on the surface of the iris are copper particles deposited during the cutting of the structure for autopsy.

SEM imaging of the inner rods showed significantly more damage to the rod surface than the iris surface, shown in Fig. 22. Because the surface magnetic field is concentrated on a small portion of the high-field sides of the inner rods, a variation in surface magnetic field intensity can be seen around the azimuth of the rods, as evidenced by decreased surface damage around the azimuth. The micrographs of the high-field rods show primarily grain formation, with some increase in surface roughness at the grain

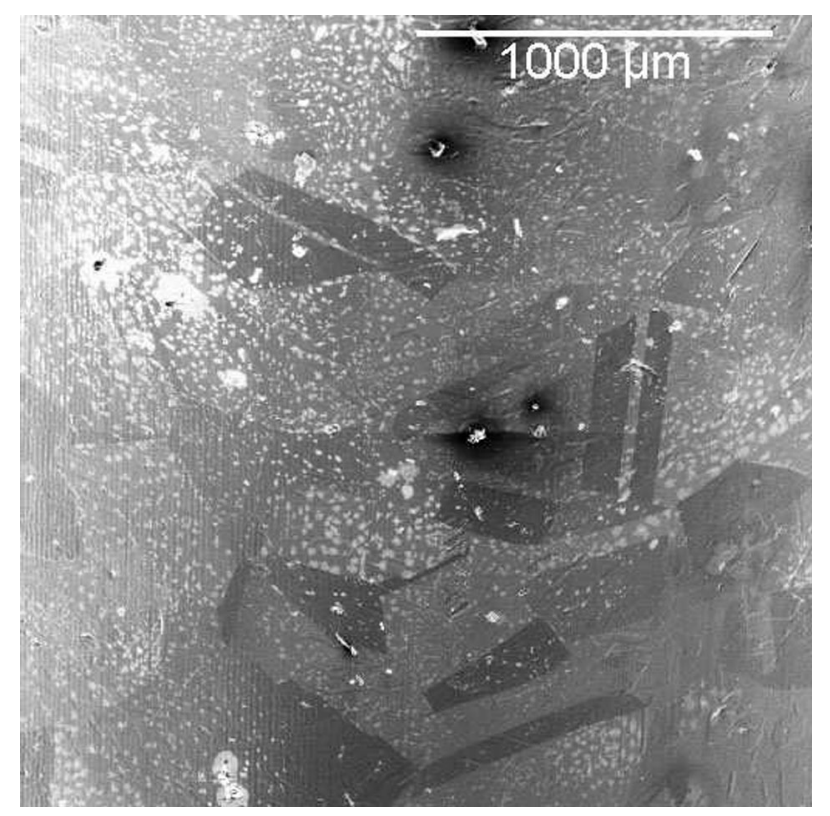

FIG. 21. SEM micrograph of damage on the iris on the input side of the PBG cell. The view shows a close-up view of the iris looking perpendicular to the axis of the structure, so that the curvature of the iris cannot be readily seen. The high-field side of this iris is to the right of the image. 


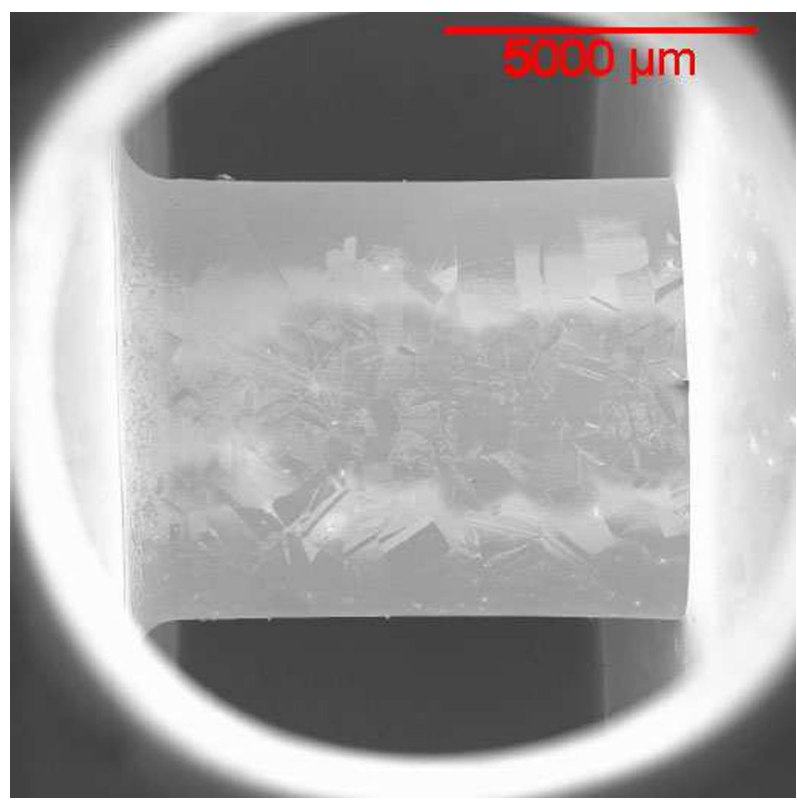

FIG. 22. SEM micrograph looking directly at high-field side of an inner rod. Grain boundaries are easily visible, and surface roughness does increase at these boundaries.

boundaries, as shown in Fig. 23. This is qualitatively different from the damage seen at the highest-field regions of the rods in the PBG-R structure, which shows an increase in surface roughness covering the entirety of the high-field side, obscuring grain boundaries in this region. The elliptical-rod PBG, in contrast, shows increased surface roughness almost exclusively at the grain boundaries, which remain visible even in the highest-field region. Because both structures saw many pulses with a peak surface temperature rise greater than $100 \mathrm{~K}$, but only the PBG-R saw excursions to over $200 \mathrm{~K}$, the hypothesis that the PBG-R suffered significant damage early in the testing as the result of many shots with extremely high peak surface temperature rise is well supported.

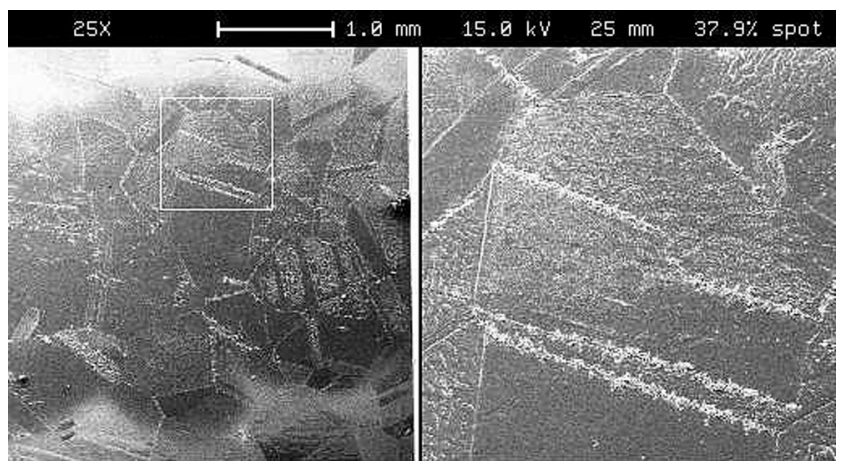

FIG. 23. Detail micrograph of the high-field side of the inner rod of the PBG-E, showing grain boundaries even at the highestfield region. The PBG-E shows significantly less damage than the PBG-R structure.

\section{DISCUSSION}

An improved PBG structure using elliptical rods in the inner row was tested at high gradient at SLAC and achieved a maximum gradient of $128 \mathrm{MV} / \mathrm{m}$ at a breakdown probability of $3.6 \times 10^{-3}$ per pulse per meter at a pulse length of $150 \mathrm{~ns}$. This is comparable to the performance of an undamped disk-loaded waveguide structure with the same iris geometry, indicating that PBG-type structures are viable for high-gradient acceleration, consistent with the goal of the revised testing methodology. This achievement of both high gradient and low breakdown probability can be attributed to improvements in both design and testing methodology. This represents a significant advance in high-gradient structure testing, as the PBG design incorporates intrinsic wakefield damping, which is lacking in the disk-loaded waveguide structures.

This result is particularly significant because the PBG-E structure has wakefield damping. The comparable performance between the PBG-E structure and the diskloaded waveguide structure indicates that the use of a PBG lattice to damp wakefields does not inherently degrade the performance of the structure. A full comparison of the PBG structure with other accelerator structures would compare the PBG-E to a structure with the same iris geometry that also has wakefield damping, and would need to consider the quality of the wakefield damping in addition to the achieved gradient at a given breakdown probability. It should be noted that the wakefield damping of the PBG-E lattice could be improved via further perturbations of the lattice, as shown in [32]. The PBG-E structure does, however, demonstrate that in principle a structure with wakefield damping via a PBG lattice can operate at high gradients.

The approximately 360 hours of testing for this structure was not long enough to evaluate the lifetime of the structure, but it can be seen that the performance did not degrade significantly during testing. If the initial operation at high gradient represented an unprocessed state that happened to perform better than the steady-state condition of the structure, then the data should show a regression to a higher breakdown probability when the structure was operated at $150 \mathrm{~ns}$ at the end of testing; this was not observed in the PBG-E testing.

The post-testing autopsy did show changes in the highfield surfaces, as well as a decrease in $Q_{0}$ of approximately $5 \%$. Both of these changes are likely the result of consistent operation of the structure above the safe threshold of $50 \mathrm{~K}$ of peak surface temperature rise in an effort to achieve higher gradients and more easily measured breakdown probabilities; when limited to a temperature of $50 \mathrm{~K}$ the breakdown rate was much too low to be reliably measured without thousands of hours of testing, something which is beyond the scope of this study. Throughout the testing the breakdown probability remained low, and the damage seen in the SEM images is qualitatively different from the 
damage observed in the high-gradient testing of the PBG-R structure, indicating that the changes in the high-field surfaces may have caused the decrease in $Q$ but were not sufficient to increase the breakdown probability. At a pulse length of 150 ns the PBG-E structure achieved a gradient of $100 \mathrm{MV} / \mathrm{m}$ and a breakdown probability of approximately $5 \times 10^{-4}$ per pulse per meter.

Future work should seek to categorize the change in the rod surface throughout testing to determine how the change in surface properties due to pulse heating correlates with changes in structure performance. The previous work by Laurent et al. [30] showed that a peak surface temperature rise of more than $50 \mathrm{~K}$ will cause changes in the surface. The PBG-E structure saw temperature rises much higher than this limit, but did not suffer significant changes in breakdown performance; this indicates that physics of rf breakdowns is more complicated than a simple pulsed heating issue. This can be studied with future PBG structures by looking at the rod surfaces in situ during testing.

Additional testing should also be done at other frequencies, e.g., $17 \mathrm{GHz}$, to determine how the achievable gradient at a given breakdown probability scales for PBG structures. Demonstration of high-frequency, highgradient, low breakdown probability operation of a structure with wakefield damping would be a very important achievement for accelerator development.

\section{ACKNOWLEDGMENTS}

Thanks to David Martin for the mechanical design of the PBG-R and PBG-E structures. The authors would also like to thank Jake Haimson and Emilio Nanni for useful discussion. This work was supported by the Department of Energy High Energy Physics, under Contract No. DEFG02-91ER40648.

[1] E. I. Smirnova, A. S. Kesar, I. Mastovsky, M. A. Shapiro, and R. J. Temkin, Phys. Rev. Lett. 95, 074801 (2005).

[2] G. R. Werner, C. A. Bauer, and J. R. Cary, Phys. Rev. ST Accel. Beams 12, 071301 (2009).

[3] R.A. Marsh, M.A. Shapiro, R. J. Temkin, V. A. Dolgashev, L. L. Laurent, J. R. Lewandowski, A. D. Yeremian, and S.G. Tantawi, Phys. Rev. ST Accel. Beams 14, 021301 (2011).

[4] C. Jing, F. Gao, S. Antipov, Z. Yusof, M. Conde, J. G. Power, P. Xu, S. Zheng, H. Chen, C. Tang et al., Phys. Rev. ST Accel. Beams 12, 121302 (2009).

[5] E. Yablonovitch, Phys. Rev. Lett. 58, 2059 (1987).

[6] S. John, Phys. Rev. Lett. 58, 2486 (1987).

[7] D. R. Smith, S. Schultz, N. Kroll, M. Sigalas, K. M. Ho, and C. M. Soukoulis, Appl. Phys. Lett. 65, 645 (1994).

[8] M. A. Shapiro, W. J. Brown, I. Mastovsky, J. R. Sirigiri, and R. J. Temkin, Phys. Rev. ST Accel. Beams 4, 042001 (2001).
[9] E. I. Smirnova, I. Mastovsky, M. A. Shapiro, R. J. Temkin, L. M. Earley, and R. L. Edwards, Phys. Rev. ST Accel. Beams 8, 091302 (2005).

[10] R. A. Marsh, M. Shapiro, R. J. Temkin, and E. I. Smirnova, in Proceedings of the 2007 Particle Accelerator Conference, Albuquerque, New Mexico (IEEE, New York, 2007), pp. 3002-3004.

[11] R. A. Marsh, M. A. Shapiro, R. J. Temkin, E. I. Smirnova, and J. F. DeFord, Nucl. Instrum. Methods Phys. Res., Sect. A 618, 16 (2010).

[12] R. A. Marsh, B. J. Munroe, M. Shapiro, and R. J. Temkin, in Proceedings of the 23rd Particle Accelerator Conference, Vancouver, Canada, 2009 (IEEE, Piscataway, NJ, 2009), WE6RFP081.

[13] V. Dolgashev, S. Tantawi, C. Nantista, Y. Higashi, and T. Higo, in Proceedings of the 2004 Linear Accelerator Conference, Lübeck, Germany, 2004 (DESY, Hamburg, 2004), pp. 766-768.

[14] V. Dolgashev, S. Tantawi, C. Nantista, Y. Higashi, and T. Higo, in Proceedings of the 21st Particle Accelerator Conference, Knoxville, 2005 (IEEE, Piscataway, NJ, 2005), pp. 595-599.

[15] V. Dolgashev, S. Tantawi, C. Nantista, Y. Higashi, and T. Higo, in Proceedings of the 2007 Particle Accelerator Conference, Albuquerque, New Mexico (Ref. [10]), pp. 2430-2432.

[16] V. Dolgashev, S. Tantawi, Y. Higashi, and T. Higo, in Proceedings of the 11th European Particle Accelerator Conference, Genoa, 2008 (EPS-AG, Genoa, Italy, 2008), pp. 742-744.

[17] V. Dolgashev, AIP Conf. Proc. 1299, 274 (2010).

[18] V. Dolgashev, S. Tantawi, Y. Higashi, and B. Spataro, in Proceedings of the 25th International Linear Accelerator Conference LINAC10, Tsukuba, Japan (KEK, Tsukuba, Japan, 2010), pp. 1043-1047.

[19] V. Dolgashev, S. Tantawi, Y. Higashi, and B. Spataro, Appl. Phys. Lett. 97, 171501 (2010).

[20] V. Dolgashev, Z. Li, S. Tantawi, A. Yeremian, Y. Higashi, and B. Spataro, in Proceedings of the 2011 International Particle Accelerator Conference, San Sebastian, Spain (EPS-AG, Spain, 2011), pp. 241-243.

[21] V. Dolgashev, S. Tantawi, A. Yeremian, Y. Higashi, and B. Spataro, in Proceedings of the 1st International Particle Accelerator Conference: IPAC'10, Kyoto, Japan (ICR, Kyoto, 2010).

[22] C. Nantista, S. Tantawi, and V. Dolgashev, Phys. Rev. ST Accel. Beams 7, 072001 (2004).

[23] B. Spataro, D. Alesini, V. Chimenti, V. Dolgashev, Y. Higashi, M. Migliorati, A. Mostacci, R. Parodi, S. Tantawi, and A. Yeremian, Nucl. Instrum. Methods Phys. Res., Sect. A 657, 88 (2011)

[24] High Frequency Structure Simulator, Ansoft Corporation [http://www.hfss.com].

[25] D. P. Pritzkau and R. H. Siemann, Phys. Rev. ST Accel. Beams 5, 112002 (2002).

[26] L.C. Maier and J.C. Slater, J. Appl. Phys. 23, 68 (1952).

[27] C. Steele, IEEE Trans. Microwave Theory Tech. 14, 70 (1966).

[28] K. Mallory and R. Miller, IEEE Trans. Microwave Theory Tech. 14, 99 (1966). 
[29] D. M. Pozar, Microwave Engineering (John Wiley \& Sons, Inc., New York, 2004).

[30] L. Laurent, S. Tantawi, V. Dolgashev, C. Nantista, Y. Higashi, M. Aicheler, S. Heikkinen, and W. Wuensch, Phys. Rev. ST Accel. Beams 14, 041001 (2011).
[31] Mathematica 8, Wolfram Research [http://www.wolfram.com].

[32] E. I. Simakov, W. B. Haynes, S. S. Kurennoy, J. F. O’Hara, E. R. Olivas, and D. Y. Shchegolkov, in Proceedings of IPAC2012, New Orleans, Louisiana, USA (IEEE, Piscataway, NJ, 2012), pp. 2801-2803. 